\title{
THE GEOMETRY OF ISOGONAL AND EQUI-TANGENTIAL SERIES*
}

\author{
BY \\ EDWARD KASNER

\section{INTRODUCTION}

We begin by considering certain simple operations on the lineal elements of the plane. A turn $T_{\alpha}$ converts each element into one having the same point and a direction making a fixed angle $\alpha$ with the original direction. By a slide $S_{k}$ the line of the element remains the same and the point moves along the line a fixed distance $k$. These transformations together generate a continuous group of three parameters $G_{3} . \dagger$ We call any transformation of this group a whirl. The only contact transformations in this group are the dilatations.

We note that the group of whirls and the group of rigid motions are isomorphic, and that the two groups are commutative, together generating an interesting six-parameter group which will be studied elsewhere. This is a sub-group of the fifteen-parameter element group converting turbines into turbines, which I introduced in 1911, itself an extension of Lie's ten-parameter contact group of higher circular geometry.

We define $\infty^{1}$ elements to be a series of elements. By applying a turn $T_{\alpha}$ to the elements of a union, we obtain a series of elements which we call an isogonal series. When a slide $S_{k}$ is applied to the elements of a union, the resulting series is called an equi-tangential series. (Finally the result of applying a whirl to the elements of a union is defined to be a whirl series. See a forthcoming Columbia dissertation by J. De Cicco, whom I wish to thank for verifying the calculations of the present paper.)

An element transformation is a correspondence between the elements of the plane. Any element transformation which transforms every union into a union is a contact transformation. We shall show that any element transformation which carries every isogonal series into an isogonal series must be the product of a conformal point transformation by a turn. Dually, any element transformation which transforms every equi-tangential series into an equi-

* Presented to the Society, September 9, 1930; received by the editors December 16, 1936.

$\dagger$ See Kasner, The group of turns and slides and the geometry of turbines, American Journal of Mathematics, vol. 33 (1911), pp. 193-202. This theory has recently been extended to spherical geometry (in an elegant synthetic treatment) by K. Strubecker, Jahresbericht der Deutschen Mathematiker Vereinigung, vol. 44 (1934), pp. 184-198, who uses the term turbine-rotation for whirl. The fact that $G_{3}$ can be generalized to any surface of constant curvature (but probably not to variable curvature) was noted in my paper, p. 193. 
tangential series must be the product of an equi-long line transformation by a magnification by a slide.

For the analytic representation of an element, it will be found convenient to use two systems of coordinates called the cartesian and the hessian coordinate systems respectively. The cartesian coordinates of an element $E$ are of course $(x, y, p)$, where $p$ is the slope of the line and $(x, y)$ are the cartesian coordinates of the point. The hessian coordinates of the element $E$ are $(u, v, w)$, where $v$ is the perpendicular distance from the origin to the line of the element $E, u$ is the angle between the perpendicular and the initial line, and $w$ is the distance from the foot of the perpendicular to the point of the element $E$.

A full discussion of the hessian system is given in Scheffer's fundamental paper (Mathematische Annalen, vol. 60 (1905)). The line $(u, v)$ and the distance $w$ are oriented.

If a general element transformation

$$
X=\phi(x, y, p), \quad Y=\psi(x, y, p), \quad P=\chi(x, y, p)
$$

is not a contact transformation it is known that it will convert exactly $\infty^{2}$ unions into unions. ${ }^{*}$ If it converts more than $\infty^{2}$, it must be a contact transformation, and thus convert all union into unions. Extensions of this theorem, involving $\infty^{3}$ and $\infty^{4}$ series of elements are found in the present paper. (See the five main theorems in italics.) In particular, it is shown that a general element transformation converts $\infty^{4}$ isogonal series into isogonal series; if more than $\infty^{4}$ arise, then all are preserved, and the resulting group of transformations is compounded of conformal (point) transformations and turns. The dual result for equi-tangential series is also valid, but demands an independent proof. There is no automatic principal of duality connecting the two theories, but only a rough analogy.

The equations of the turn $T_{\alpha}$ in cartesian coordinates are

$$
X=x, \quad Y=y, \quad \Theta=\theta+\alpha,
$$

where $\theta$ denotes the inclination of the element; and hence the equations of any isogonal series are

$$
y=y(x), \quad \arctan p=\arctan y^{\prime}(x)+\arctan \alpha,
$$

where $\alpha$ is the constant angle.

The equations of the slide $S_{k}$ in hessian coordinates are

$$
U=u, \quad V=v, \quad W=w+k,
$$

* See Kasner, The general transformation theory of differential elements, American Journal of Mathematics, vol. 32 (1910), p. 393. 
and hence the equations of any equi-tangential series are

$$
v=v(u), \quad w=v^{\prime}(u)+k,
$$

where $k$ is the constant distance.

A turbine is a special case of both isogonal series and equi-tangential series where the base curve is a circle. All whirls convert turbines into turbines. The total group of turbines involves fifteen parameters. Lie's ten-parameter group converts circles into turbines.

PART I. IsOgonal SERIES AND THE CONFORMAL GROUP

We now prove our theorems about element transformations.

THEOREM 1. Under any element transformation there exists a three-parameter family of unions which are carried into isogonal series. Any element transformation which carries every union into an isogonal series must be the product of a contact transformation by a turn.

By the element transformation

$$
X=\phi(x, y, p), \quad Y=\psi(x, y, p), \quad P=\chi(x, y, p),
$$

the elements of a union*

$$
y=y(x), \quad p=y^{\prime}(x),
$$

become the elements

$$
X=\phi\left(x, y, y^{\prime}\right), \quad Y=\psi\left(x, y, y^{\prime}\right), \quad P=\chi\left(x, y, y^{\prime}\right) .
$$

If this series is an isogonal series, then we must have

$$
\arctan \chi=\arctan \frac{d \psi}{d \phi}+\text { constant }
$$

Differentiating (1) with respect to $x$, we obtain

$$
\frac{d}{d x}\left(\frac{\frac{d \psi}{d x}}{\frac{d \phi}{d x}}\right)=\frac{\frac{d \chi}{d x}}{1+\chi^{2}}\left[1+\left(\frac{\frac{d \psi}{d x}}{\frac{d \phi}{d x}}\right)^{2}\right] .
$$

Now let $f$ denote the fraction, involving $x, y, y^{\prime}, y^{\prime \prime}$,

* This representation omits point-unions, but we can verify the validity of our main theorems by a separate discussion without difficulty. 


$$
f=\frac{\frac{d \psi}{d x}}{\frac{d \phi}{d x}}=\frac{\psi_{x}+y^{\prime} \psi_{y}+y^{\prime \prime} \psi_{y^{\prime}}}{\phi_{x}+y^{\prime} \phi_{y}+y^{\prime \prime} \phi_{y^{\prime}}}
$$

Then from (2) and (3), we obtain

$$
\frac{d f}{d x}=\frac{\chi_{x}+y^{\prime} \chi_{y}+y^{\prime \prime} \chi_{y^{\prime}}}{1+\chi^{2}}\left[1+f^{2}\right]
$$

The equation (4) is a differential equation in $y$ and the highest order of $y$ in this equation is the third order. For the coefficient of $y^{\prime \prime \prime}$ in the expanded equation is given by

$$
\left(\phi_{x}+y^{\prime} \phi_{y}\right) \psi_{y^{\prime}}-\left(\psi_{x}+y^{\prime} \psi_{y}\right) \phi_{y^{\prime}},
$$

which, in general, is not zero. Thus, it follows that this differential equation has three parameters in its complete solution and therefore there exists a three-parameter family of unions which by the given element transformation are carried into isogonal series.

We now determine all the element transformations which carry every union into an isogonal series. Then the condition (4) where $f$ is given by (3) must hold for every union. Thus (4) must be an identity in $x, y, y^{\prime}, y^{\prime \prime}, y^{\prime \prime \prime}$. The coefficient of $y^{\prime \prime \prime}$ must then be zero, so that

$$
\left(\phi_{x}+y^{\prime} \phi_{y}\right) \psi_{y^{\prime}}-\left(\psi_{x}+y^{\prime} \psi_{y}\right) \phi_{y^{\prime}}=0 .
$$

Again (5) must be an identity in $x, y, y^{\prime}$. Hence

$$
\frac{\psi_{x}+p \psi_{y}}{\phi_{x}+p \phi_{y}}=\frac{\psi_{p}}{\phi_{p}}
$$

From (6) we obtain

$$
f=\frac{\psi_{x}+y^{\prime} \psi_{y}+y^{\prime \prime} \psi_{y^{\prime}}}{\phi_{x}+y^{\prime} \phi_{y}+y^{\prime \prime} \phi_{y^{\prime}}}=\frac{\psi_{x}+y^{\prime} \psi_{y}}{\phi_{x}+y^{\prime} \phi_{y}}=\frac{\psi_{y^{\prime}}}{\phi_{y^{\prime}}}=\lambda\left(x, y, y^{\prime}\right) .
$$

To determine $\lambda$, substitute it into equation (4) so that

$$
\frac{\lambda_{x}+y^{\prime} \lambda_{y}+y^{\prime \prime} \lambda_{y^{\prime}}}{1+\lambda^{2}}=\frac{\chi_{x}+y^{\prime} \chi_{v}+y^{\prime \prime} \chi_{v^{\prime}}}{1+\chi^{2}}
$$

This is an identity. Hence

$$
\frac{\lambda_{y^{\prime}}}{1+\lambda^{2}}=\frac{\chi_{y^{\prime}}}{1+\chi^{2}}, \quad \frac{\lambda_{x}+y^{\prime} \lambda_{y}}{1+\lambda^{2}}=\frac{\chi_{x}+y^{\prime} \chi_{y}}{1+\chi^{2}} .
$$


From these two equations, it follows immediately, that

$$
\arctan \lambda=\arctan \chi+\alpha, \quad(\alpha \text { constant }) .
$$

From (6), (7), and (8) we obtain

$$
\frac{\psi_{x}+p \psi_{y}}{\phi_{x}+p \phi_{y}}=\frac{\psi_{p}}{\phi_{p}}=\tan (\arctan \chi+\alpha) .
$$

From (9), we obtain our result. So Theorem 1 is proved.

We next study the transformation of isogonal series into isogonal series.

THEOREM 2. Under any element transformation there exists a four-parameter family of isogonal series which are carried into isogonal series. If more than $\infty$ such series exist then all $\infty^{\infty}$ isogonal series exist. Any element transformation which carries every isogonal series into an isogonal series must be the product of a conformal point transformation by a turn. These transformations form a group.

The proof is rather complicated and will occupy the next three pages. Systems of partial differential equations appear, which fortunately can be integrated in finite form.

Under any element transformation, the elements of an isogonal series

$$
y=y(x), \quad \arctan p=\arctan y^{\prime}(x)+\arctan k,
$$

become the series

$$
X=\phi(x, y, p), \quad Y=\psi(x, y, p), \quad P=\chi(x, y, p) .
$$

Now if this series is an isogonal series, we must have

$$
\frac{d}{d x}\left(\frac{\frac{d \psi}{d x}}{\frac{d \phi}{d x}}\right)=\frac{\frac{d \chi}{d x}}{1+\chi^{2}}\left[1+\left(\frac{\frac{d \psi}{d x}}{\frac{d \phi}{d x}}\right)^{2}\right]
$$

Let $f$ again denote the fraction

$$
f=\frac{\frac{d \psi}{d x}}{\frac{d \phi}{d x}}=\frac{\psi_{x}+y^{\prime} \psi_{y}+p^{\prime} \psi_{p}}{\phi_{x}+y^{\prime} \phi_{y}+p^{\prime} \phi_{p}} .
$$

Since $\arctan y^{\prime}=\arctan p-\arctan k$, we have from (11)

$$
f=\frac{(1+k p) \psi_{x}+(p-k) \psi_{y}+(1+k p) p^{\prime} \psi_{p}}{(1+k p) \phi_{x}+(p-k) \phi_{y}+(1+k p) p^{\prime} \phi_{p}} .
$$


From (10) we obtain

$$
\frac{d f}{d x}=\frac{\frac{d \chi}{d x}}{1+\chi^{2}}\left[1+f^{2}\right]
$$

Equation (13) is a differential equation in $y$, and the highest order of $y$ in this equation is the third order. For the coefficient of $y^{\prime \prime \prime}$ is the coefficient of $p^{\prime \prime}$

$$
(1+k p)\left[\left\{(1+k p) \phi_{x}+(p-k) \phi_{y}\right\} \psi_{p}-\left\{(1+k p) \psi_{x}+(p-k) \psi_{y}\right\} \phi_{p}\right],
$$

multiplied by the coefficient of $y^{\prime \prime \prime}$ in $p^{\prime \prime}$. Since the coefficient of $y^{\prime \prime \prime}$ in $p^{\prime \prime}$ in general is not zero, it follows that the coefficient of $y^{\prime \prime \prime}$ in (13) in general is not zero. Therefore the complete solution of (13) will contain three constants of integration and the constant $k$ of the original isogonal series. Thus, we see that there exists a four-parameter family of isogonal series which transform into isogonal series. This proves the first part of our theorem.

We now determine all the element transformations which carry every isogonal series into an isogonal series. Since a union is a special type of isogonal series, it follows that any element transformation which changes every isogonal series into an isogonal series must be the product of a contact transformation by a turn. Then from the group properties of the set of turn transformations, it follows that the contact transformation must carry every isogonal series into an isogonal series. Hence, we must find all the contact transformations which transform every isogonal series into an isogonal series.

We shall show that the contact transformation must be a point transformation. If this were not the case, then we should have

$$
X=\phi(x, y, p), \quad Y=\psi(x, y, p), \quad P=\chi(x, y, p)=\frac{\psi_{x}+p \psi_{y}}{\phi_{x}+p \phi_{y}}=\frac{\psi_{p}}{\phi_{p}},
$$

where $\phi_{p} \neq 0$, and $\psi_{p} \neq 0$. Now equation (13) must be an identity in $x, y, p, p^{\prime}$, $p^{\prime \prime}$ and $k$. Hence the coefficient of $p^{\prime \prime}$ must be zero so that

$(1+k p)\left[\left\{(1+k p) \phi_{x}+(p-k) \phi_{y}\right\} \psi_{p}-\left\{(1+k p) \psi_{x}+(p-k) \psi_{y}\right\} \phi_{p}\right]=0$.

Since $1+k p \neq 0$, we obtain

(14) $\left[\left(\phi_{x}+p \phi_{y}\right) \psi_{p}-\left(\psi_{x}+p \psi_{y}\right) \phi_{p}\right]+k\left[\left(p \phi_{x}-\phi_{y}\right) \psi_{p}-\left(p \psi_{x}-\psi_{y}\right) \phi_{p}\right]=0$.

Since (14) is an identity in $k$, we have

$$
\left(\phi_{x}+p \phi_{y}\right) \psi_{p}-\left(\psi_{x}+p \psi_{y}\right) \phi_{p}=0, \quad\left(p \phi_{x}-\phi_{y}\right) \psi_{p}-\left(p \psi_{x}-\psi_{y}\right) \phi_{p}=0,
$$

and therefore we obtain 


$$
\frac{\psi_{x}}{\phi_{x}}=\frac{\psi_{y}}{\phi_{y}}=\frac{\psi_{p}}{\phi_{p}} .
$$

But (15) makes the jacobian of the transformation vanish. This means that the only contact transformations of the desired type are point transformations.

We now find all the point transformations

$$
X=\phi(x, y), \quad Y=\psi(x, y), \quad P=\frac{\psi_{x}+p \psi_{y}}{\phi_{x}+p \phi_{y}},
$$

which carry every isogonal series into an isogonal series. Then the equation (13) must be an identity where

$$
\chi=\frac{\psi_{x}+p \psi_{y}}{\phi_{x}+p \phi_{y}}, \quad f=\frac{(1+k p) \psi_{x}+(p-k) \psi_{y}}{(1+k p) \phi_{x}+(p-k) \phi_{y}} .
$$

In (13) we then can set the coefficient of $p^{\prime}$ equal to zero so that

$$
\begin{array}{r}
\frac{\left[(1+k p) \phi_{x}+(p-k) \phi_{y}\right]\left(k \psi_{x}+\psi_{y}\right)-\left[(1+k p) \psi_{x}+(p-k) \psi_{y}\right]\left(k \phi_{x}+\phi_{y}\right)}{\left[(1+k p) \phi_{x}+(p-k) \phi_{y}\right]^{2}} \\
=\frac{\chi_{p}}{1+\chi^{2}}\left[1+\left\{\frac{(1+k p) \psi_{x}+(p-k) \psi_{y}}{(1+k p) \phi_{x}+(p-k) \phi_{y}}\right\}^{2}\right] .
\end{array}
$$

From (16) and (17) we obtain

$$
\begin{array}{r}
{\left[(1+k p) \phi_{x}+(p-k) \phi_{y}\right]\left[k \psi_{x}+\psi_{y}\right]-\left[(1+k p) \psi_{x}+(p-k) \psi_{y}\right]\left(k \phi_{x}+\phi_{y}\right)} \\
{\left[(1+k p) \phi_{x}+(p-k) \phi_{y}\right]^{2}+\left[(1+k p) \psi_{x}+(p-k) \psi_{y}\right]^{2}} \\
=\frac{\left(\phi_{x}+p \phi_{y}\right) \psi_{y}-\left(\psi_{x}+p \psi_{y}\right) \phi_{y}}{\left(\phi_{x}+p \phi_{y}\right)^{2}+\left(\psi_{x}+p \psi_{y}\right)^{2}}
\end{array}
$$

which, upon simplification, becomes

$$
\begin{array}{r}
\frac{\left[\left(\phi_{x}+p \phi_{y}\right)+k\left(p \phi_{x}-\phi_{y}\right)\right]\left(k \psi_{x}+\psi_{y}\right)-\left[\left(\psi_{x}+p \psi_{y}\right)+k\left(p \psi_{x}-\psi_{y}\right)\right]\left(k \phi_{x}+\phi_{y}\right)}{\left[\left(\phi_{x}+p \phi_{y}\right)+k\left(p \phi_{x}-\phi_{y}\right)\right]^{2}+\left[\left(\psi_{x}+p \psi_{y}\right)+k\left(p \psi_{x}-\psi_{y}\right)\right]^{2}} \\
=\frac{\phi_{x} \psi_{y}-\psi_{x} \phi_{y}}{\left(\phi_{x}+p \phi_{y}\right)^{2}+\left(\psi_{x}+p \psi_{y}\right)^{2}} .
\end{array}
$$

Since (18) is an identity in $k$, we can set the coefficient of $k^{2}$ equal to zero so that

$$
\frac{\psi_{x}\left(p \phi_{x}-\phi_{y}\right)-\phi_{x}\left(p \psi_{x}-\psi_{y}\right)}{\left(p \phi_{x}-\phi_{y}\right)^{2}+\left(p \psi_{x}-\psi_{y}\right)^{2}}=\frac{\phi_{x} \psi_{y}-\psi_{x} \phi_{y}}{\left(\phi_{x}+p \phi_{y}\right)^{2}+\left(\psi_{x}+p \psi_{y}\right)^{2}}
$$


and thus we obtain

$$
\frac{-\psi_{x} \phi_{y}+\phi_{x} \psi_{y}}{\left(p \phi_{x}-\phi_{y}\right)^{2}+\left(p \psi_{x}-\psi_{y}\right)^{2}}=\frac{\phi_{x} \psi_{y}-\psi_{x} \phi_{y}}{\left(\phi_{x}+p \phi_{y}\right)^{2}+\left(\psi_{x}+p \psi_{y}\right)^{2}} .
$$

Since the jacobian $\phi_{x} \psi_{y}-\psi_{x} \phi_{y} \neq 0$, we have

$$
\left(\phi_{x}+p \phi_{y}\right)^{2}+\left(\psi_{x}+p \psi_{y}\right)^{2}=\left(p \phi_{x}-\phi_{y}\right)^{2}+\left(p \psi_{x}-\psi_{y}\right)^{2} .
$$

Now (20) is an identity in $p$ so that we have

$$
\phi_{x}^{2}+\psi_{x}^{2}=\phi_{y}^{2}+\psi_{y}^{2}, \quad \phi_{x} \phi_{y}+\psi_{x} \psi_{y}=0 .
$$

These equations are then equivalent to the Cauchy-Riemann equations (together with the conjugate equations)

$$
\phi_{y}= \pm \psi_{x}, \quad \phi_{x}=\mp \psi_{y} .
$$

Hence the point transformation is conformal.

The sufficiency of our result is obvious so that Theorem 2 is completely proved.

Part II. Equi-tangential SERIES AND the EQUi-LONG GROUP

THEOREM 3. Under any element transformation there exists a three-parameter family of unions which are carried into equi-tangential series. Any element transformation which carries every union into an equi-tangential series must be the product of a contact transformation by a slide.

By any element transformation

$$
U=\phi(u, v, w), \quad V=\psi(u, v, w), \quad W=\chi(u, v, w),
$$

the elements of a union*

$$
v=v(u), \quad w=v^{\prime}(u),
$$

become the elements

$$
U=\phi\left(u, v, v^{\prime}\right) ; \quad V=\psi\left(u, v, v^{\prime}\right), \quad W=\chi\left(u, v, v^{\prime}\right) .
$$

If this series is an equi-tangential series, then

$$
\chi=\frac{d \psi}{d \phi}+\text { constant }
$$

and hence we obtain

\footnotetext{
* We are assuming that the union is not a straight line union. The final results are, however, true in all cases.
} 


$$
\frac{d}{d u}\left(\frac{\psi_{u}+v^{\prime} \psi_{v}+v^{\prime \prime} \psi_{v^{\prime}}}{\phi_{u}+v^{\prime} \phi_{v}+v^{\prime \prime} \phi_{v^{\prime}}}-\chi\right)=0 .
$$

Now (21) is a differential equation in $v$ and the highest order of $v$ is the third order. For the coefficient of $v^{\prime \prime \prime}$ is

$$
\left(\phi_{u}+v^{\prime} \phi_{v}\right) \psi_{v^{\prime}}-\left(\psi_{u}+v^{\prime} \psi_{v}\right) \phi_{v^{\prime}},
$$

which in general is not zero. Hence the complete solution of the differential equation (21) contains three constants of integration, and therefore it is seen that there exists a three-parameter family of unions which are transformed into equi-tangential series. If more than $\infty^{3}$ unions of this kind exist, then all $\infty^{\infty}$ unions must be of this kind.

We now determine all the element transformations which carry every union into an equi-tangential series. Then (21) must be an identity in $u$, $v$, $v^{\prime}, v^{\prime \prime}, v^{\prime \prime \prime}$. Thus the coefficient of $v^{\prime \prime \prime}$ in (21) must be zero. That is,

$$
\left(\phi_{u}+v^{\prime} \phi_{v}\right) \psi_{v^{\prime}}-\left(\psi_{u}+v^{\prime} \psi_{v}\right) \phi_{v^{\prime}}=0 .
$$

This is an identity. Hence

$$
\frac{\psi_{u}+w \psi_{v}}{\phi_{u}+w \phi_{v}}=\frac{\psi_{w}}{\phi_{w}}
$$

Then we obtain

$$
\frac{\psi_{u}+v^{\prime} \psi_{v}+v^{\prime \prime} \psi_{v^{\prime}}}{\phi_{u}+v^{\prime} \phi_{v}+v^{\prime \prime} \phi_{v^{\prime}}}=\frac{\psi_{u}+v^{\prime} \psi_{v}}{\phi_{u}+v^{\prime} \phi_{v}}=\frac{\psi_{v^{\prime}}}{\phi_{v^{\prime}}}=\lambda\left(u, v, v^{\prime}\right) .
$$

To determine $\lambda$, substitute it into (21) and we obtain

$$
\frac{d}{d u}(\lambda-\chi)=0
$$

This immediately yields the result

$$
\lambda(u, v, w)=\chi(u, v, w)+\alpha, \quad(\alpha \text { constant }) .
$$

From (22), (23), and (24) we obtain

$$
\frac{\psi_{u}+w \psi_{v}}{\phi_{u}+w \phi_{v}}=\frac{\psi_{w}}{\phi_{w}}=\chi+\alpha .
$$

Interpreting equations (25), we obtain Theorem 3.

THEOREM 4. Under any element transformation there exists a four-parameter family of equi-tangential series which are carried into equi-tangential series. Any element transformation which carries every equi-tangential series into an equi- 
tangential series must be the product of an equi-long transformation (every two collinear elements are transformed into collinear elements in such a way that the distance between them is preserved), by a magnification by a slide. These transformations form a group.

We observe that this group is generated by three partial groups (the infinite equi-long group depending on arbitrary functions of $u+j v$, where $j^{2}=0$, slides, and magnifications); and is thus larger than the dual group of Theorem 2 which is generated by two partial groups (conformal and turns).

We proceed now to the rather long proof of Theorem 4. By any element transformation, the equi-tangential series

$$
v=v(u), \quad w=v^{\prime}(u)+k,
$$

becomes the series

$$
U=\phi(u, v, w), \quad V=\psi(u, v, w), \quad W=\chi(u, v, w) .
$$

Now if this series is an equi-tangential series, then we must have

$$
\frac{d}{d u}\left(\frac{\psi_{u}+v^{\prime} \psi_{v}+w^{\prime} \psi_{w}}{\phi_{u}+v^{\prime} \phi_{v}+w^{\prime} \phi_{w}}-\chi\right)=0,
$$

and hence

$$
\frac{d}{d u}\left(\frac{\psi_{u}+(w-k) \psi_{v}+w^{\prime} \psi_{w}}{\phi_{u}+(w-k) \phi_{v}+w^{\prime} \phi_{w}}-\chi\right)=0 .
$$

The equation (26) is a differential equation in $v$ and the highest order of $v$ in (26) is the third order. For the coefficient of $v^{\prime \prime \prime}$ is

$$
\left[\phi_{u}+(w-k) \phi_{v}\right] \psi_{w}-\left[\psi_{u}+(w-k) \psi_{v}\right] \phi_{w},
$$

which in general is not zero. Therefore the complete solution of (26) contains three constants of integration and the constant $k$ of the original equi-tangential series. Thus, it is seen that there exists a four-parameter family of equi-tangential series which are transformed into equi-tangential series.

We now determine all the element transformations which carry every equi-tangential series into an equi-tangential series. Since a union is a special type of equi-tangential series, it follows that any element transformation which carries every equi-tangential series into an equi-tangential series must, by Theorem 3, be the product of a contact transformation by a slide. From this it follows that we must find all contact transformations by which every equi-tangential series corresponds to an equi-tangential series.

We shall show that the contact transformation is a line transformation. If this were not the case, then 


$$
U=\phi(u, v, w), \quad V=\psi(u, v, w), \quad W=\chi(u, v, w)=\frac{\psi_{u}+w \psi_{v}}{\phi_{u}+w \phi_{v}}=\frac{\psi_{w}}{\phi_{w}},
$$

where $\phi_{w} \neq 0$ and $\psi_{w} \neq 0$. The equation (26) now must be an identity in $u, v$, $w, k, w^{\prime}, w^{\prime \prime}$. Hence the coefficient of $w^{\prime \prime}$ must be zero

$$
\left[\phi_{u}+(w-k) \phi_{v}\right] \psi_{w}-\left[\psi_{u}+(w-k) \psi_{v}\right] \phi_{w}=0 .
$$

Since (27) is an identity, we have

$$
\left(\phi_{u}+w \phi_{v}\right) \psi_{w}-\left(\psi_{u}+w \psi_{v}\right) \phi_{w}=0, \quad \phi_{v} \psi_{w}-\psi_{v} \phi_{w}=0,
$$

and hence

$$
\frac{\phi_{u}}{\psi_{u}}=\frac{\phi_{v}}{\psi_{v}}=\frac{\phi_{w}}{\psi_{w}}
$$

But (28) makes the jacobian of the transformation vanish. This means that the only possible contact transformations of the desired kind are line transformations.

Let us now suppose that our contact transformation has the properties just stated. Its equations are

$$
U=\phi(u, v), \quad V=\psi(u, v), \quad W=\frac{\psi_{u}+w \psi_{v}}{\phi_{u}+w \phi_{v}}
$$

Then (26) becomes

$$
\frac{d}{d u}\left[\frac{\psi_{u}+(w-k) \psi_{v}}{\phi_{u}+(w-k) \phi_{v}}-\chi\right]=0,
$$

where

$$
\chi=\frac{\psi_{u}+w \psi_{v}}{\phi_{u}+w \phi_{v}}
$$

Since (29) is an identity, the coefficient of $w^{\prime}$ is zero

$$
\frac{\left[\phi_{u}+(w-k) \phi_{v}\right] \psi_{v}-\left[\psi_{u}+(w-k) \psi_{v}\right] \phi_{v}}{\left[\phi_{u}+(w-k) \phi_{v}\right]^{2}}=\chi_{w} .
$$

Simplifying this last equation and taking account of (30), we have

$$
\frac{\phi_{u} \psi_{v}-\psi_{u} \phi_{v}}{\left[\phi_{u}+(w-k) \phi_{v}\right]^{2}}=\frac{\phi_{u} \psi_{v}-\psi_{u} \phi_{v}}{\left(\phi_{u}+w \phi_{v}\right)^{2}} .
$$

Since $\phi_{u} \psi_{v}-\psi_{u} \phi_{v} \neq 0$, we then obtain 


$$
\left[\phi_{u}+(w-k) \phi_{v}\right]^{2}=\left[\phi_{u}+w \phi_{v}\right]^{2} .
$$

Now (31) is an identity. Hence

$$
\phi_{v}=0
$$

so that we have

$$
\phi=F(u)
$$

Substituting (32) into (29) we obtain

$$
\frac{d}{d u}\left[\frac{\psi_{u}+(w-k) \psi_{v}}{F^{\prime}(u)}-\frac{\psi_{u}+w \psi_{v}}{F^{\prime}(u)}\right]=0 .
$$

Now (33) is an identity. Hence the coefficient of $k$ must be zero:

$$
\frac{d}{d u} \frac{\psi_{v}}{F^{\prime}(u)}=0
$$

and therefore

$$
F^{\prime}(u)\left[\psi_{v u}+v^{\prime} \psi_{v v}\right]-\psi_{v} F^{\prime \prime}(u)=0 .
$$

The equation (34) is an identity. Hence

$$
F^{\prime}(u) \psi_{v u}-\psi_{v} F^{\prime \prime}(u)=0, \quad F^{\prime}(u) \psi_{v v}=0 .
$$

From equations (32) and (35) we obtain

$$
\left\{\begin{array}{l}
U=F(u), \\
V=m v F^{\prime}(u)+G(u), \\
W=m w+m v \frac{F^{\prime \prime}(u)}{F^{\prime}(u)}+\frac{G^{\prime}(u)}{F^{\prime}(u)},
\end{array}\right.
$$

where $F(u)$ and $G(u)$ are functions of $u$ only and where $m$ is a constant. This transformation is the product of an equi-long line transformation by a magnification.

The sufficiency of the result is obvious so that our Theorem 4 is completely proved.

From Theorems 2 and 4 we easily obtain the following result:

THEOREM 5. Any element transformation which carries every isogonal series into an isogonal series and every equi-tangential series into an equi-tangential series must be the product of a rigid motion by a magnification. We thus obtain the similitude group. 
In conclusion we remark that the family of $\infty^{2}$ unions converted into unions by an arbitrary element transformation is of entirely general character; but that the three-parameter and four-parameter manifolds considered in Theorems 1-4 are of special character. The geometric properties of these manifolds will be considered elsewhere. See abstracts in the Bulletin of the American Mathematical Society, vol. 36 (1930), pp. 353 and 487.

Columbia University, NEW YORK, N. Y. 